\title{
Superradiant Amplification of Acoustic Beams via Medium Rotation
}

\author{
D. Faccio ${ }^{1,2}$ and E. M. Wright ${ }^{2}$ \\ ${ }^{1}$ School of Physics \& Astronomy, University of Glasgow, G12 8QQ Glasgow, United Kingdom \\ ${ }^{2}$ College of Optical Sciences, University of Arizona, Tucson, Arizona 85721, USA
}

(Received 4 September 2018; revised manuscript received 3 November 2018; published 23 July 2019)

\begin{abstract}
Superradiant gain is the process in which waves are amplified via their interaction with a rotating body, examples including the evaporation of a spinning black hole and electromagnetic emission from a rotating metal sphere. In this Letter we elucidate how superradiance may be realized experimentally in the field of acoustics, and predict the possibility of nonreciprocally amplifying or absorbing acoustic beams carrying orbital angular momentum by propagating them through an absorbing medium that is rotating. We discuss a possible geometry for realizing acoustic superradiant amplification using existing technology.
\end{abstract}

DOI: 10.1103/PhysRevLett.123.044301

Introduction.-Prior to Hawking's epoch-making discovery that black holes must shrink and eventually explode when the quantum vacuum is accounted for [1], in 1969 Penrose argued that it must be possible to extract energy from the rotational motion of a spinning black hole $[2,3]$. This idea was then further developed in 1971 by Zel'dovich who argued that spinning black holes must spontaneously evaporate, counter to understanding based on vacuum solutions of general relativity [4]. In a tour de force of physical intuition Zel'dovich argued this assertion based on the analog system of a spinning metallic sphere in which incident vacuum fluctuations of the electromagnetic field, composed of virtual photons with energy equal to the zeropoint value, are converted to real photons, the energy being drawn from the rotational energy of the sphere. In subsequent work, Zel'dovich and co-workers showed using quantum electrodynamics $[5,6]$ that a metallic cylinder bathed in the electromagnetic vacuum must radiate electromagnetic energy, leading to slowing of the rotation of the cylinder [7,8]. Zel'dovich argued that what applies to the electromagnetic vacuum fluctuations surrounding a spinning conducting medium must also apply to the gravitational vacuum fluctuations, or virtual gravitons, surrounding a black hole, so that spinning black holes must radiate [4]. Misner suggested that if real gravitational waves (as opposed to the virtual waves associated with vacuum fluctuations) are incident on a spinning black hole they will be amplified [9], a process that he referred to as superradiance [10].

Since these seminal works, it has become clear that the complex of ideas surrounding radiation from rotating bodies bathed in the quantum vacuum (now referred to as the Zel'dovich effect), or the related amplification of real waves incident on a rotating absorbing body (now referred to as superradiance), are quite generic as speculated by Zel'dovich and can apply to such disparate wave systems as gravity [9-11], nonlinear optics [12-14], matter waves for cold atoms [15-17], including also hydrodynamical wave amplification in nonhomogeneous flows [18]. We also underline the recent demonstration of superradiant amplification in water where incident waves were reflected and amplified from a draining vortex [19].

Our goal in this Letter is to elucidate how the process of superradiance may be realized and studied in the field of acoustics, with the key prediction that it is possible to nonreciprocally amplify or absorb acoustic beams carrying orbital angular momentum by propagating them through an absorbing medium that is rotating. In this context we note that in comparison to previous treatments of superradiance in which inward propagating radial waves are amplified upon reflection from the outer surface of a rotating cylinder $[4-7,20]$. Here, we consider the transmission of an incident beam through the rotating medium. Our proposal is timely in view of the recent experimental observation of negative frequencies for acoustic waves carrying orbital angular momentum that are detected using a pair of rotating microphones [21]: such negative frequencies signal that the so-called Zel'dovich-Misner condition is satisfied, which is a prerequisite for observing superradiant gain [7].

Governing equations.-We consider acoustic waves propagating predominantly along the $z$ axis in an isotropic and absorbing medium that is rotating around the $z$ axis at frequency $\Omega$. Our starting point is the wave equation in the rotating frame for the density variations $\tilde{\rho}(\mathbf{r}, t)$ with respect to the background value $\rho_{0} \gg|\tilde{\rho}|$ [22-24]. Then, following the notation of Ref. [24], the wave equation in the presence of acoustic absorption is

$$
\frac{\partial^{2} \tilde{\rho}}{\partial t^{2}}-\Gamma^{\prime} \nabla^{2} \frac{\partial \tilde{\rho}}{\partial t}-v^{2} \nabla^{2} \tilde{\rho}=0
$$

where $v$ is the velocity of sound and $\Gamma^{\prime}$ is the damping parameter. To proceed, we first transfer to the nonrotating laboratory frame in cylindrical coordinates $\mathbf{r}=(r, z, \phi)$ using the change of variables $[4,7]$ 


$$
t^{\prime}=t, \quad r^{\prime}=r, \quad z^{\prime}=z, \quad \phi^{\prime}=\phi+\Omega t .
$$

Then, performing a change of variables to the nonrotating (primed) frame, and dropping the primes for simplicity in notation, we obtain

$$
\left(\frac{\partial}{\partial t}+\Omega \frac{\partial}{\partial \phi}\right)^{2} \tilde{\rho}-\Gamma^{\prime} \nabla^{2}\left(\frac{\partial}{\partial t}+\Omega \frac{\partial}{\partial \phi}\right) \tilde{\rho}-v^{2} \nabla^{2} \tilde{\rho}=0
$$

This wave equation in the laboratory frame is the basis for the following analysis.

Bessel beams.-Our analysis is based on the fact that Eq. (3) has exact Bessel beam solutions that carry orbital angular momentum (OAM) $[25,26]$. In particular, we consider an acoustic wave of winding number $\ell$ and frequency $\omega>0$, and in complex representation the exact solution is

$$
\tilde{\rho}(r, z, \phi, t)=A J_{|\ell|}\left(k_{r} r\right) e^{i\left(\ell \phi-\omega t+k_{z} z\right)} .
$$

Here, $k_{r}$ is the radial component of the acoustic wave vector and $k_{z}$ the $z$ component. We choose $k_{z}$ (see below) such that its real part $\Re\left(k_{z}\right)$ is greater than zero, in which case the solution Eq. (4) represents a Bessel beam propagating along the positive $z$ axis.

Dispersion relation.-Substituting the exact solution (4) into the acoustic wave equation Eq. (3), in the laboratory frame we obtain the exact dispersion relation

$$
k_{r}^{2}+k_{z}^{2}=\frac{(\omega-\ell \Omega)^{2} / v^{2}}{1-i \Gamma^{\prime}(\omega-\ell \Omega) / v^{2}},
$$

the solution of which may be written as

$$
\frac{k_{z}}{k_{0}}= \pm \sqrt{\frac{[(\omega-\ell \Omega) / \omega]^{2}}{1-i \eta[(\omega-\ell \Omega) / \omega]}-\frac{k_{r}^{2}}{k_{0}^{2}}},
$$

where $\eta=\Gamma^{\prime} \omega / v^{2}$ is a positive dimensionless parameter characterizing the acoustic absorption per length $1 / k_{0}$, and $k_{0}=\omega / v$. To use Eq. (6), we choose a value for $k_{r}<k_{0}$, and Eq. (5) is then solved for $k_{z}$ : as stated above, we choose the sign in the solution such that $\Re\left(k_{z}\right)>0$. The absorption experienced by the acoustic field is proportional to the imaginary part $\Im\left(k_{z}\right)$, and as a measure of this we consider the absorption per length $1 / k_{0}$ :

$$
\frac{\alpha}{k_{0}}=\frac{\Im\left(k_{z}\right)}{k_{0}} .
$$

Another key quantity is the phase velocity $v_{p}=\omega / \Re\left(k_{z}\right)$, and it is useful to consider the phase velocity scaled to $v=\omega / k_{0}$,
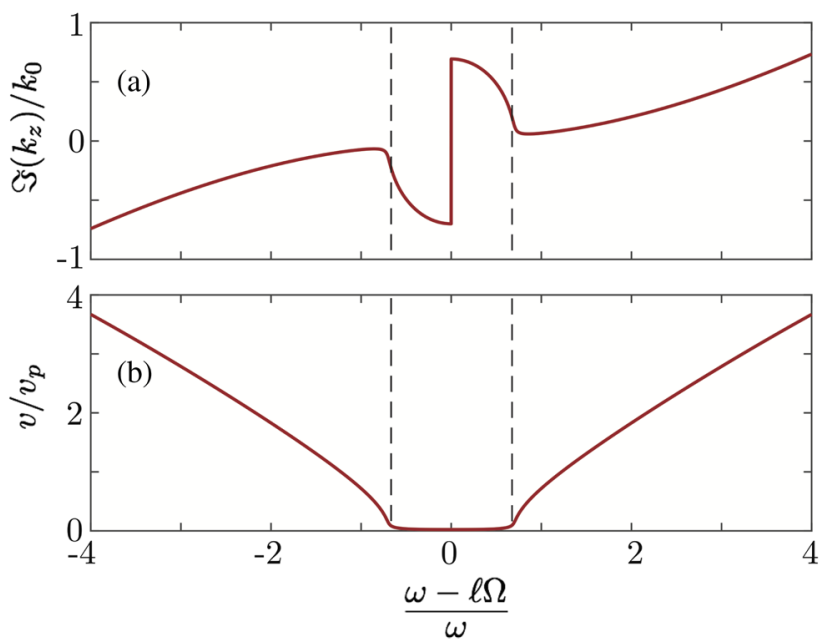

FIG. 1. Plots of (a) the absorption $\Im\left(k_{z}\right) / k_{0}$ and (b) the scaled inverse phase velocity $v / v_{p}=\Re\left(k_{z}\right) / k_{0}$ both as functions of $(\omega-\ell \Omega) / \omega$, and for parameters $\eta=0.1$ and $k_{r} / k_{0}=0.7$. The vertical dashed lines delineate the range in Eq. (9). Negative $\Im\left(k_{z}\right) / k_{0}$ implies gain.

$$
\frac{v_{p}}{v}=\frac{k_{0}}{\Re\left(k_{z}\right)} .
$$

A supersonic phase velocity arises if $v_{p} / v>1$, and a subsonic phase velocity if $v_{p} / v<1$.

We now examine the dispersion relation searching for conditions under which superradiant amplification can arise for individual Bessel beams. Since a general input beam can be viewed as a wave packet of Bessel beams, the insights so gained will apply to more general incident acoustic beams.

Superradiant amplification.-The solutions involved here generally have nonzero values for both the real and imaginary part of $k_{z}$ and as such they represent propagating wave solutions [as opposed to evanescent waves that have $\Re\left(k_{z}\right)=0$ ]. Thus, in our case with $\Re\left(k_{z}\right)>0$, the condition for gain is $\Im\left(k_{z}\right)<0$.

As an illustration of the superradiant amplification that can arise, Fig. 1 shows plots of (a) the scaled absorption $\Im\left(k_{z}\right) / k_{0}$ and (b) the scaled inverse phase velocity $v / v_{p}=\Re\left(k_{z}\right) / k_{0}$, both as functions of $(\omega-\ell \Omega) / \omega$, and for parameters $\eta=0.1$ and $k_{r} / k_{0}=0.7$. What is striking from Fig. 1(a) is that the scaled absorption is negative for $(\omega-\ell \Omega)<0$ signifying gain, with the transition from absorption to gain occurring in the range

$$
-\frac{k_{r}}{k_{0}}<\frac{(\omega-\ell \Omega)}{\omega}<\frac{k_{r}}{k_{0}},
$$

which is delineated by the vertical dashed lines, and maximum scaled gain $-\Im\left(k_{z}\right) / k_{0}=k r / k_{0}$ in this range. Physically, the energy needed for this superradiant amplification is drawn from the rotational energy of the medium, 
which means that energy must be supplied to keep the medium rotating at the constant rate $\Omega$ or the medium would spin down.

Based on the above analysis, the condition for superradiant amplification is

$$
\omega-\ell \Omega<0,
$$

which coincides with the Zel'dovich-Misner condition for superradiant gain [7], and corresponds to when the rotationally shifted frequency of the source in the rotating frame becomes negative. Accompanying the superradiant amplification in the range Eq. (9) we find that the inverse scaled phase velocity shown in Fig. 1(b) is supersonic: the scaled phase velocity $v_{p} / v$ is infinite at $(\omega-\ell \Omega)=0$, and significantly larger than $v$ over the rest of the range. Another way to look at this is to note that $\Re\left(k_{z}\right)=0$ for $(\omega-\ell \Omega)=0$, implying an acoustic impedance $Z$ that is close to zero in the range in Eq. (9). We can then talk of a $Z$-near-zero (ZNZ) regime, in analogy to epsilon-near-zero (ENZ) physics in optics [27] and note that the two regimes share many common aspects that are related to the nature of wave propagation in the presence of a vanishing impedance. Both ENZ optics and ZNZ acoustics exhibit a series of signatures such as the aforementioned large or infinite phase velocity, a concomitant lengthening to infinity of the wavelength and a close-to-zero group velocity [28]. The ZNZ point at which $(\omega-\ell \Omega)=0$ cannot be accessed due to the extreme consequences of a zero-group velocity (or infinite phase velocity and purely dc, nonoscillatory nature of the wave) that imply a complete reflection of the incoming wave. Therefore, the discontinuity from absorption to gain at $(\omega-\ell \Omega)=0$, although not ruled out from thermodynamic considerations [7], is not a problem as it is not physically accessible. Nevertheless, in analogy with ENZ studies in optics, we can still couple waves into the medium in the ZNZ regime (away from the $Z=0$ point) and observe enhanced acoustic interactions.

We note that significant absorption and gain occur also outside the range given by Eq. (9). This is illustrated in Fig. 1(a) that shows absorption, $(\omega-\ell \Omega)>0$, and gain, $(\omega-\ell \Omega)<0$, outside the range in Eq. (9) and with accompanying phase velocities that can be subsonic or supersonic, see Fig. 1(b).

We also remark that in the gain region for $\omega<\ell \Omega$ the corresponding group velocity becomes negative, the phase velocity remaining positive: This leads to the acoustic analog of superluminal pulse propagation that has been studied extensively in optics in which a pulse incident on the medium is transmitted but with a negative delay, meaning that the net energy flow remains in the direction set by the phase velocity $[29,30]$.

Nonreciprocity.-We consider the backward propagating version of Eq. (4)

$$
\tilde{\rho}_{b}(r, z, \phi, t)=A J_{|\ell|}\left(k_{r} r\right) e^{i\left(-\ell \phi-\omega t-k_{z} z\right)},
$$

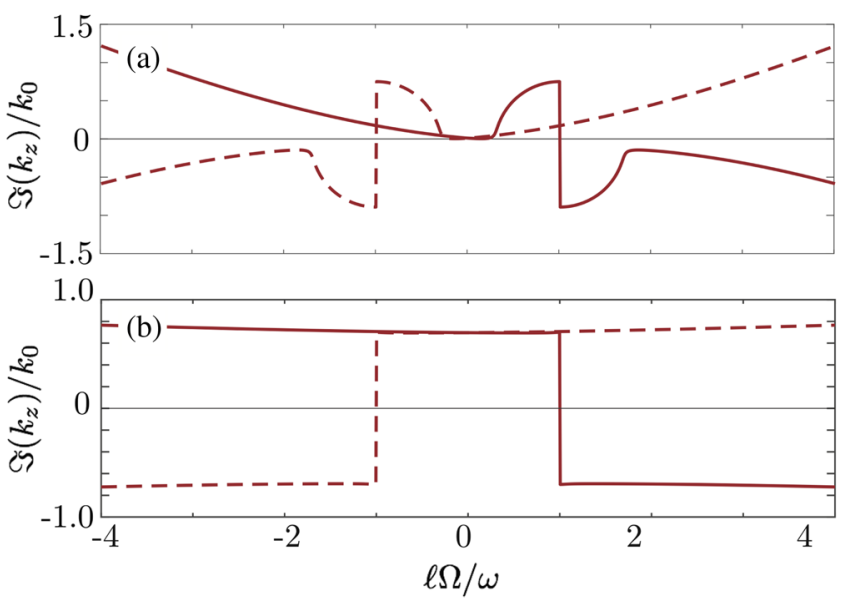

FIG. 2. Scaled absorption $\Im\left(k_{z}\right) / k_{0}$ versus $\ell \Omega / \omega$ for the forward (solid line) and backward (dashed line) propagating Bessel beam solutions, and parameters $k_{r} / k_{0}=0.7$ (chosen to match the conditions of Ref. [21]) and (a) $\eta=0.1$; (b) $\eta=10$.

where $k_{z}$ is again chosen such that its real part $\Re\left(k_{z}\right)$ is greater than zero, in which case the solution (11) represents a Bessel beam propagating along the negative $z$ axis. Here, $+\ell$ has been replaced by $-\ell$ so that sense of rotation of the OAM is preserved with the change of propagation direction. Then for propagation along the negative $z$ axis the corresponding solution of the dispersion relation is

$$
\frac{k_{z}}{k_{0}}=\sqrt{\frac{[(\omega+\ell \Omega) / \omega]^{2}}{1-i \eta[(\omega+\ell \Omega) / \omega]}-\frac{k_{r}^{2}}{k_{0}^{2}}} .
$$

Since the form of $k_{z}$ for the counterpropagating waves in Eqs. (6) and (12) differ by the factors $(\omega \pm \ell \Omega)$ this means that for a fixed rotation rate counterpropagating acoustic beams with the same OAM can experience different gain or absorption. That is, there is nonreciprocity with respect to propagation direction that can be used to realize sound isolation [31,32].

An example of this nonreciprocity is shown in Fig. 2(a) where the scaled absorption $\Im\left(k_{z}\right) / k_{0}$ is shown as a function of $\ell \Omega / \omega$ for the forward (solid line) and backward (dashed line) propagating Bessel beam solutions, and parameters $\eta=0.1$ and $k_{r} / k_{0}=0.7$. This figure has several interesting features: first, if we consider a given propagation direction (solid or dashed line) then for a fixed rotation rate $\Omega$ it is clear that winding numbers $\pm \ell$ experience different absorptions, and this highlights the dichroism for acoustic OAM alluded to earlier. Second, in the range $|\ell \Omega / \omega|<1$ both directions of propagation experience absorption but one more than the other: This highlights the nonreciprocity discussed above, and if the differential absorption times length product can be made large enough this can be used to realize sound isolation. Third, in the complementary range $|\ell \Omega / \omega|>1$ one direction of propagation experiences gain while the other 


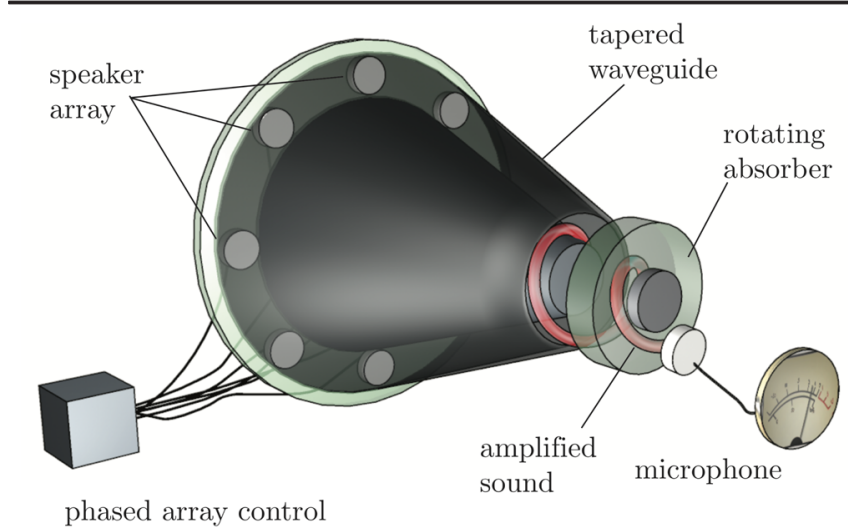

FIG. 3. Schematical representation of a possible experimental layout that would allow us to measure superradiant gain by extraction of energy from the rotation of an absorbing disk.

experiences absorption. To the best of our knowledge this is a case never discussed before [31,32].

Experimental considerations.-One of the key features associated with superradiant gain is the change in sign of the material absorption from positive to negative that occurs when the rotational-Doppler shifted frequency becomes negative. Such a negative-frequency condition was observed recently in the experiments reported by Gibson et al. [21] with a microphone on a platform rotating at $\sim 10-50 \mathrm{~Hz}$ and placed in the path of a (nonrotating) acoustic wave source with $90 \mathrm{~Hz}$ oscillation frequency (in the laboratory frame) and OAM $\ell=4$. In these experiments, the inversion of the OAM sign, or temporal inversion of the beam occurs when $\omega-\ell \Omega<0$, thus clearly setting the scene for observing superradiant gain given that the key condition Eq. (10) can be satisfied in experiments. However, in order to observe gain one must extend the experiment by adding absorption: this can be simply achieved by placing an absorbing layer in front of the microphones. In Fig. 3 we show an example of a possible experimental layout that would satisfy the conditions used in our analysis. Generation of the acoustic OAM beam follows the same scheme as in [21]: the speakers form a phase array of sine-wave emitters. By increasing the acoustic sine-wave phase from one speaker to the next, full phase shifts of multiples $\ell$ of $2 \pi$ will give rise to acoustic OAM beams that are then directed towards the rotating absorbing disk via a hollow waveguide. A microphone is placed behind this disk that measures the transmitted sound intensity. The signature of superradiant gain would be a crossover from absorption to gain as the rotation speed is increased and Eq. (10) is satisfied. We note that gain or, phrased differently, an increase in phonon number will not depend on the reference frame in which it is measured. It is therefore possible to either use microphones that are stationary in the lab frame or corotating solidly with the disk, as in [21]. This invariance of the gain with respect to the measurement reference frame can be directly verified starting from Eq. (1) and performing the analysis in the corotating frame.

It also worth noting that the acoustic wavelength will typically be an order of magnitude or more greater than the dimensions of the overall experiment and more than 2 orders of magnitude larger than the absorbing disk thickness. The waveguide structure therefore guides the wave towards the absorbing disk [21] where, in this deeply subwavelength regime, a maximum of 50\% absorption can be expected for a stationary medium $[33,34]$. Standard acoustic absorbing foam will typically absorb $\sim 10 \%$ at $100 \mathrm{~Hz}$ (for a $1 \mathrm{~cm}$ thick slab) and would correspond to the case $\eta \sim 10$, see Fig. 2(b). From Fig. 2(b) one can infer that existing technology is predicted to give rise to an acoustic gain of order $1 \%-10 \%$ and should therefore be readily observable. In the Supplemental Material, we provide a more in-depth analysis of the acoustic gain for a Bessel beam incident from air onto a rotating subwavelength medium [35].

We conclude by noting that in our analysis we tacitly assumed that the rotating medium is a solid as opposed to a fluid, which precludes mechanical vorticity arising in the medium due to the rotation. One may still consider the problem of vorticity in the surrounding air medium but this too is reduced to a minimum in the range of frequencies tested so far up to $50 \mathrm{~Hz}$. We also have experimental evidence that vorticity is not a problem in our regime, as this would appear as a strong noise in any measurements. But such noise is not seen in the experiments performed by Gibson et al. [21], which indeed served as a starting point for the parameter space explored in our work.

Conclusions. - Superradiant amplification in the form of amplification from a spinning absorber is very generic and was first discovered in the context of spinning black holes. Yet, it has not been observed to date due to the difficulty in satisfying the required conditions, in particular mechanical rotation frequencies that are close to the wave frequencies. Here, we provide details of an acoustic system that could realistically realize such conditions with currently available experimental setups. Moreover, our analysis has unveiled new physical effects, i.e., nonreciprocal absorption and gain and an enhanced superradiant gain that occurs within a specific window of mechanical rotation frequencies, Eq. (9), corresponding to the $Z$-near-zero, $Z N Z$, regime. In this region the group velocity is close to zero and the enhanced gain can be ascribed to "slow wave" effects, i.e., to an increase in the mode density, which enhances interaction with the medium, in analogy with slow light absorption and gain in optics [36,37]. We expect experiments in the near future to provide the first evidence of acoustic superradiance, i.e., the increase of acoustic wave energy at the expense of mechanical rotational energy of an absorbing medium.

The authors thank Professor Miroslav Kolesik and Professor Masud Mansuripur of the College of Optical Sciences at the University of Arizona and Professor 
Graham Gibson, Professor Ermes Toninelli, and Professor Miles Padgett at the University of Glasgow for useful discussions and suggestions regarding this work. D.F. acknowledges financial support from EPSRC (U.K., Grant No. EP/P006078/2).

[1] S. W. Hawking, Nature (London) 248, 30 (1974).

[2] R. Penrose, Riv. Nuovo Cimento, Numero Speziale I, 257 (1969); Gen. Relativ. Gravit. 34, 1141 (2002).

[3] R. Penrose and B. M. Floyd, Nature (London) 229, 177 (1971).

[4] Y. B. Zel'dovich, Pis'ma Zh. Eksp. Teor. Fiz. 14, 270 (1971) [JETP Lett. 14, 180 (1971)].

[5] Y. B. Zel'dovich, Zh. Eksp. Teor. Fiz. 62, 2076 (1972) [Sov. Phys. JETP 35, 1085 (1972)].

[6] Ya. B. Zel'dovich, L. V. Rozhanskii, and A. A. Starobinskii, Izv. Vyssh. Uchebn. Zaved., Radiofiz. 29, I008 (1986).

[7] J. D. Bekenstein and M. Schiffer, Phys. Rev. A 58, 064014 (1998).

[8] M. F. Maghrebi, R. L. Jaffe, and M. Kardar, Phys. Rev. Lett. 108, 230403 (2012).

[9] C. W. Misner, Phys. Rev. Lett. 28, 994 (1972).

[10] C. W. Misner, Bull. Am. Phys. Soc. 17, 472 (1972).

[11] R. Brito, V. Cardoso, and P. Pani, Superradiance, Lecture Notes in Physics 906 (Springer, New York, 2015).

[12] F. Marino, Phys. Rev. A 78, 063804 (2008).

[13] F. Marino, M. Ciszak, and A. Ortolan, Phys. Rev. A 80, 065802 (2009).

[14] D. Faccio and E. M. Wright, Phys. Rev. Lett. 118, 093901 (2017).

[15] F. Federici, C. Cherubini, S. Succi, and M. P. Tosi, Phys. Rev. A 73, 033604 (2006).

[16] H. Takeuchi, M. Tsubota, and G. Volovik, J. Low Temp. Phys. 150, 624 (2008).

[17] N. Ghazanfari and O. E. Mustecaplioglu, Phys. Rev. A 89, 043619 (2014).
[18] L. A. Ostrovskii, S. A. Rybak, and L. Sh, Tsimring Usp. Fiz. Nauk 150, 417 (1986).

[19] T. Torres, S. Patrick, A. Coutant, M. Richartz, E. W. Tedford, and S. Weinfurtner, Nat. Phys. 13, 833 (2017).

[20] V. Cardoso, A. Coutant, M. Richartz, and S. Weinfurtner, Phys. Rev. Lett. 117, 271101 (2016).

[21] G. M. Gibson et al., Proc. Natl. Acad. Sci. U.S.A. 115, 3800 (2018).

[22] J. W. S. Rayleigh, Theory of Sound (Dover, New York, 1896/1945), Vol. 2.

[23] H. Lamb, Hydrodynamics (Cambridge University Press, Cambridge, England, 1932).

[24] R. W. Boyd Nonlinear Optics, 3rd ed. (Academic Press, Amsterdam, 2008), Chap. 8.

[25] D. Baresch, J.-L. Thomas, and R. Marchiano, Phys. Rev. Lett. 116, 024301 (2016).

[26] N. Jimenez, R. Pico, V. Sanchez-Morcillo, V. RomeroGarcia, L. M. Garcia-Raffi, and K. Staliunas, Phys. Rev. E 94, 053004 (2016).

[27] I. Liberal and N. Engheta, Nat. Photonics 11, 149 (2017).

[28] K. L. Tsakmakidis, O. Hess, R. W. Boyd, and Z. Zhang, Science 358, eaan5196 (2017).

[29] K. T. McDonald, Am. J. Phys. 69, 607 (2001).

[30] M. Mitchell and R. Y. Chiao, Am. J. Phys. 66, 14 (1998).

[31] R. Fleury, D. L. Sounas, C. F. Siek, M. R. Haberman, and A. Alu, Science 343, 516 (2014).

[32] Z. Yang, F. Gao, X. Shi, X. Lin, Z. Gao, Y. Chong, and B. Zhang, Phys. Rev. Lett. 114, 114301 (2015).

[33] S. Thongrattanasiri, F. H. L. Koppens, and F. J. Garcia de Abajo, Phys. Rev. Lett. 108, 047401 (2012).

[34] T. Roger et al., Nat. Commun. 6, 7031 (2015).

[35] See Supplemental Material at http://link.aps.org/ supplemental/10.1103/PhysRevLett.123.044301 for an indepth theoretical analysis that accounts for the air-mediumair boundary conditions.

[36] L. O'Faolain et al., Opt. Express 18, 27627 (2010).

[37] S. Ek, P. Lunnemann, Y. Chen, E. Semenova, K. Yvind, and J. Mork, Nat. Commun. 5, 5039 (2014). 